\title{
An epidemiological model of the corn stunt system in Central America
}

\author{
John Vandermeer \\ Department of Biology, University of Michigan, Ann Arbor, MI 48109, U.S.A. \\ and Alison Power \\ Section of Ecology and Systematics, Cornell University, Ithaca, NY, U.S.A.
}

(Accepted 8 February 1990)

\begin{abstract}
Vandermeer, J. and Power, A., 1990. An epidemiological model of the corn stunt system in Central America. Ecol. Modelling, 52: 235-248.

There is increasing appreciation on the part of ecologists of the crucial role that pathogens may play in the structure of plant populations and communities. This study used classic equations of epidemiology to analyze corn stunt in Central America, which includes a leafhopper, Dalbulus maidis, a spiroplasma, and corn, Zea mays. Given the underlying structure, the system is expected always to tend to a $100 \%$ infection rate of the corn. The prospect of artificially raising the death rate of infected corn, that is, the selective destruction of infected corn plants or highly infected fields, was investigated using the model. The destruction of infected plants must be greater as the transmission probabilities increase, as the disappearance rate of the leafhopper decreases, and as the number of leafhoppers per plant decreases. Parameters of the model were estimated using data from Nicaragua and Costa Rica. To control the disease by eliminating infected corn plants would require elimination of between $0.11 \%$ and $8.3 \%$ of the population per week in Nicaragua; the corresponding figures for Costa Rica were $2.2 \%$ to $>100 \%$.
\end{abstract}

\section{INTRODUCTION}

The ecology and epidemiology of disease has recently received a great deal of theoretical attention (Anderson and May, 1979, 1982; May and Anderson, 1979; Anderson, 1981), most of which has been confined to animal diseases. In the past simple exponential or logistic models have been used to describe the progress curves of plant diseases (e.g. Vanderplank, 1963; Zadoks and Schein, 1979; Thresh, 1983; Burdon, 1987), and recently 
progress has been made in more mechanistic modeling disease dynamics, especially with reference to the spread of a disease from a focus (Jeger, 1983; Minogue and Fry, 1983a,b; Van den Bosch et al., 1988a,b,c). However, to date no one has attempted to modify classic epidemiological models for use with either endemic or epidemic plant diseases involving an intermediate vector. Yet there is increasing appreciation on the part of ecologists of the crucial role that pathogens may play in the structure of plant populations and communities (Leonard and Czochor, 1978; Burdon, 1982, 1987; Hamilton, 1982; Dinoor and Eshed, 1984; Ellstrand and Antonovics, 1985). We propose that the classic epidemiological approach usually applied to human and other animal diseases could be extremely illuminating with regard to the dynamics of plant diseases.

Perhaps the most studied epidemiological system involving two hosts is schistosomiasis, one reference citing over 10000 papers on the subject (Warren and Newill, 1967). Despite the intense attention this system had received, according to Cohen, "To date no mathematical model of the disease has come close to representing faithfully most of what is known about the natural history of the disease" (Cohen, 1976). In this light it is perhaps not surprising to encounter a less studied system for which no useful mathematical model has yet been developed and tested. The paucity of appropriate theoretical work has been particularly acute for the group of plant pathogens transmitted by insects, a group including most viral diseases and many of the most economically important plant diseases. Yet the structure of the classic epidemiological models may be quite appropriate for this group of pathogens, since these models are often derivatives of the original Ross malaria models which involve both host and vector populations. In this paper we modify recent epidemiological theory to apply to a particular pathogen-vector-host plant system with which one of us has worked extensively (Power, 1985, 1987, 1988). The objective is to explore the potential use of classic epidemiology models, appropriately modified, as an aid in understanding the dynamics of insect-vectored plant diseases, using the system of corn stunt in Central America as a case study. In addition to the goal of understanding the ecology of insect vectored plant diseases in general, this particular disease system is the cause of dramatic yield losses in corn throughout much of Central and South America, suggesting that there may be practical consequences of the research.

\section{DISEASE SYSTEM}

In Central America, maize production is severely limited by the corn stunt disease which is incited by a spiroplasma (Davis, 1974). This pathogen causes severe stunting and can result in total loss of a crop, although its 
more typical effect is simply a dramatic yield loss. Symptoms include shortening of the internodes, the development of axillary shoots, male sterility, chlorotic striping of the leaves, and in some corn varieties, pronounced leaf reddening.

There are three seasons of maize production, two traditional ones - the primera and postrera - and one recent one of less extent, irrigation. The primera occurs at the begining of the wet season (and thus can vary from year to year and place to place) approximately May or June. The postrera begins at the end of the canicula, or 'little dry season', usually in August. Maize production under irrigation can be initiated at any time between late December to mid-February. Thus, viewing the system broadly, there are three 'flushes' in the biomass of the primary host, with a predictably low period during the later portion of the dry season.

The spiroplasma can be transmitted to maize only by leafhopper feeding and is not transovarially transmitted or seed-borne. Although several leafhopper species are capable of transmitting the corn stunt spiroplasma, the most important vector throughout Latin America is the corn leafhopper Dalbulus maidis Delong and Wolcott (Homoptera: Cicadellidae, Deltocephalinae). When $D$. maidis individuals are given access to the pathogen, nearly 100\% are capable of transmitting it (Markham and Alivizatos, 1983), but neither the longevity nor reproduction of $D$. maidis are detectably affected by the infection (Madden et al., 1984). D. maidis is a Zea specialist, reproducing only on maize (Zea mays) and related species (mainly the teosintes) (Nault and Delong, 1980; Nault, 1983; Nault et al. 1983; Nault and Madden, 1985). Because the teosintes do not occur outside Mexico and Guatemala, corn is the only viable host plant for D. maidis throughout most of its range.

The spiroplasma is transmitted in a persistent fashion, requiring a latency period before becoming circulative in either the insect vector or the host plant, and is retained for long periods by the vector (Nault, 1980). It appears that relatively long feeding periods (hours to days) by the vector are required for acquisition and transmission (Anaya Garcia, 1974), as is commonly the case for persistent pathogens. There is a specific latency period in the plant before the disease can be transmitted to another vector individual, depending on temperature and the maize variety (Martinez-Lopez, 1976; Nault, 1980).

This disease system offers several advantages for evaluating the utility of classic epidemiological models for understanding the dynamics of plant diseases. First, it is a relatively simple agricultural system in which the components are well-known and can be monitored easily; second, the pathogen can be transmitted only by vector feeding; and third, in most areas there is a single species of vector which is a feeding specialist on the host plant. 
Elementary malaria model. Noting that the system is mathematically similar to the malaria system, we begin with the classic model of Ross (1911). Thus, if $I_{1}$ is the proportion of the human population infected, $I_{2}$ the proportion of the mosquito population infected, $b$ is the biting rate of the mosquito, $f_{1}$ and $f_{2}$ are the proportions of the infected individuals that are infectious, $\mu_{1}$ is the recovery rates of the human population, $\mu_{2}$ the death rate of the mosquito population, and $N_{1}$ and $N_{2}$ are the population densities of humans and mosquitoes, respectively, we have:

$\mathrm{d} I_{1} / \mathrm{d} t=\left(N_{1} / N_{2}\right) b f_{2} I_{2}\left(1-I_{1}\right)-\mu_{1} I_{1}$

$\mathrm{d} I_{2} / \mathrm{d} t=b f_{1} I_{1}\left(1-I_{2}\right)-\mu_{2} I_{2}$

Converting the variables to their meaning in the current context we have $I_{1}$ representing the proportion of infected corn plants, $I_{2}$ the proportion of infected leafhoppers, $N_{1}$ and $N_{2}$ the respective population densities, $b$ is the 'biting rate' of the leafhopper $1, f_{1}$ and $f_{2}$ are the proportions of the infected individuals that are infectious, $\mu_{1}$ is the recovery rate plus the death rate of the corn (Ross assumed that the recovery rate of humans, the corn analogue in the model, was large relative to the death rate), and $\mu_{2}$ the disappearance rate of the leafhoppers. Lotka's (1923) analysis of these equations has been summarized numerous times (Anderson, 1981; MacDonald, 1957; Liu, 1988; Bailey, 1975), and it is a well-established result that the so-called disease reproductive rate determines the qualitatively distinct dynamic outcomes of the system. Disease reproductive rate is given as:

$R=\left(N_{1} / N_{2}\right) b^{2} f_{2} f_{1} / \mu_{1} \mu_{2}$

When $R \leq 1$, the system is stable, with the equilibrium at $I_{1}=I_{2}=0$, thus the effective rate will decline until equilibrium is reached at zero. When $R>1$ the system is unstable at the origin (i.e. $I_{1}=I_{2}=0$ ) but has a stable equilibrium and positive values of both $I_{1}$ and $I_{2}$, thus will increase until it stabilizes eventually.

In the particular case of the Dalbulus-spiroplasma-corn system, the death rate of corn, due to the spiroplasma, is virtually nil, the problem with the disease being stunting and yield reduction, not the loss of entire plants. Thus $\mu_{1}$ must be very small, making $R \geq 1$ almost always the case. Given this assumption, the equilibrium values of the two proportions are given as: $I_{1}^{*}=1, I_{2}^{*}=b f_{1} /\left(b f_{1}+\mu_{2}\right)$, whence we see that the system is always expected to tend to a $100 \%$ infection rate of the corn (this is simply because in the classic malaria model a key force maintaining less than full human infection is the recovery rate). 
Prospects for controlling the disease through destruction of infected corn plants or fields. The prospect of artificially elevating $\mu_{1}$, which in the present context would correspond to the selective destruction (roguing) of infected corn plants (or, at a regional level, whole corn fields), is logical, but to our knowledge is not currently done in actual productive practice. What might be the prospects of a program in which whole corn fields were burned based on a region-wide program of disease detection program or in which individual producers were encouraged to eliminate diseased parts of their fields at some specific rate? We take as a goal the reduction of disease reproductive rate to less than unity. We thus require:

$\mu_{1}>N_{1} b^{2} f_{1} f_{2} / N_{2} \mu_{2}$

The values for all of these parameters can be approximated from the data of Power (1987). The way we have cast the problem, the well-known quandary of lags in transmission from vector to host as well as from host to vector, are agglomerated into a combination of $b, f_{1}$, and $f_{2}$. Several time-related phenomena are involved in these functions. The vector must feed for some time period [absolute minimum thought to be about 1/2 h (Anaya Garcia, 1974)]. Once infected, the spiroplasma must incubate inside the corn for a time period of 16-46 days (Martinez-Lopez, 1976; Nault, 1980). Once infected with the spiroplasma, the vecor can retransmit only after an incubation period of 17-23 days (Nault, 1980). It may be interesting and even ultimately necessary to model these time lag phenomena directly. For example Aron and May (1982) explicitly studied the incubation period in malarial transmission, because they were interested in questions related directly to possible changes in that incubation period. In contrast, our interest at this point is in the overall dynamics of the disease system, allowing the incubation phenomena to remain as constants. It is thus, in our opinion, more reasonable to model such phenomena as part of the general parameters $f_{1}$ and $f_{2}$.

Interpreting $f_{1}$ and $f_{2}$ as probabilities is probably the most convenient formulation, which implicitly includes the time lags involved in various incubation periods. Suppose, then, that $f_{2}$ is the probability that an infected leafhopper feeding on a corn plant will transmit the disease to the corn plant, we must ask what is the probability that an individual remains $1 / 2 \mathrm{~h}$ or more. While we have no quantitative estimate of this figure, from personal observations it seems that at least two of the three plants encountered by an average leafhopper in a day are fed upon for more than $1 / 2 \mathrm{~h}$. Thus we set the lower limit on the probability of transmission as $2 / 3$, such that the approximate range of $f_{2}$ is $0.67-1.0$. The other parameter, $f_{1}$, is the probability that a leafhopper feeding on an infected corn plant will acquire the spiroplasma. This formulation includes the fact that the leaf- 
hopper may not feed long enough to pick up the infection, and that the infection may not have yet been incubated long enough within the corn plant so as to have made the corn plant infectious. The average life span of a corn plant is about 90 days, which suggests that the median time of infection is at 45 days. With an incubation period of 16-46 days, and with an average (median) infection incidence at 45 days, the probability that an infected plant is not in its transmissible state is somewhere between $16 / 45$ and $46 / 45$. Add to this the probability of $1 / 2 \mathrm{~h}$ or more of feeding (see above) and we compute the probability of an infected corn plant being infective as somewhere between 0.24 and 0.68 .

For notational convenience we replace the term $b f_{1}$ with $b_{1}$ and $b f_{2}$ with $b_{2}$, and call $b_{1}$ and $b_{2}$ the transmission probabilities. Equation (2) then becomes:

$\mu_{1}>b_{1} b_{2} /\left[\mu_{2}\left(N_{2} / N_{1}\right)\right]$

whence we see qualitatively that the destruction of infected corn plants must be greater as the transmission probabilities increase, as the disappearance rate of the leafhopper decreases, and as the number of leafhoppers per plant decreases. The latter point is a surprising and, on first glance, a non-intuitive result. Its significance can perhaps be made clear with an examination of the equilibrium form of the equation for corn infection. We have, at equilibrium:

$I_{1}^{*}=\left[b_{1} b_{2}-\mu_{1} \mu_{2}\left(N_{2} / N_{1}\right)\right]\left[b_{1} b_{2}+\mu_{1} b_{1}\left(N_{2} / N_{1}\right)\right]^{-1}$

Equation (3) is graphed in Fig. 1 for three different values of $N_{2}$. When the system is characterized by large leafhopper densities, the equilibrium infection rate can be brought down from its very high value at small $\mu_{1}$, to a very low value with only a small increase in $\mu_{1}$ (Fig. 1 ). In a system with low leafhopper densities the method of artificially introducing a higher value of $\mu_{1}$ will have less of an impact; which is to say, the value of $\mu_{1}$ must be elevated quite high to achieve similar reductions in the incidence of the disease. This would suggest that in regions in which leafhopper densities are normally high, such as in the northern Pacific plain of Nicaragua, the method of destroying infected corn fields might be more effective than in areas of naturally low leafhopper densities such as Costa Rica (Power, unpublished data).

The value of $\mu_{2}$ in the present context must not only include the death rate of the leafhoppers but also the net balance between immigration into the area and migration out of the area. The most appropriate measure of $\mu_{2}$ is the 'disappearance rate', which represents disappearances both by death and movement out of an area. This parameter has been directly measured for one population in Nicaragua (Power, 1987). 


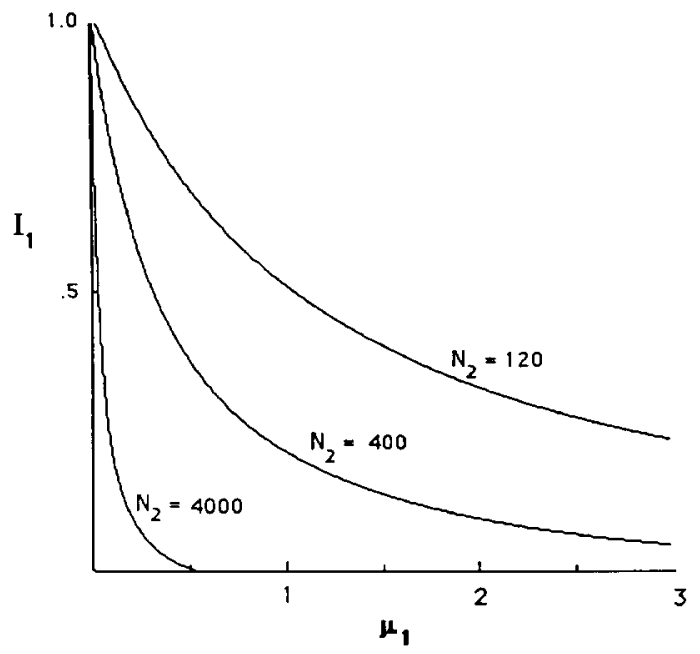

Fig. 1. Graph of equation (3), the equilibrium infection of corn $\left(I_{1}\right)$ as a function of death rate $\left(\mu_{1}\right)$, for various values of $N_{2}$, the population density of leafhoppers. Base parameters are $b_{1}=2, b_{2}=0.35, \mu_{2}=0.13, N_{1}=400$.

The parameter $N_{2} / N_{1}$ is highly variable, as discussed above. Several values are available from the literature and from our own unpublished data (Gadea and Power, 1984; Power, 1987, 1988; unpublished data). The values of $N_{1}$ can vary considerably since they represent a parameter that is under human control, but for purposes of this model all that is required is an estimate of the ratio of $N_{2}$ to $N_{1}$.

Various estimates of these parameters are presented in Table 1. Using the extreme values in the table we can calculate a critical value of $\mu_{1}$ (see equation 2) that ranges from 0.062 to 4.74 in Nicaragua and from 1.25 to 158.14 in Costa Rica, for a reduction of the disease incidence to zero. These figures represent the number of corn plants per day per standard plot, which in this case is set by our estimate of $\mu_{2}$, and is a plot of $75 \mathrm{~m}^{2}$ containing 400 plan is [the size of the experimental plots in Power (1988)]. Thus we translate these figures into $0.11 \%$ of the population per week to $8.3 \%$ of the population per week in Nicaragua, and the corresponding figures of 2.2 to $>100 \%$ (actually $277 \%$, clearly not logically possible) in Costa Rica. The range of these estimates is especially large for the Costa Rican data. If the lower estimate were more nearly correct, we might suggests a cultural regime of destroying infected corn fields at a rate that would provide for the elimination of $2.2 \%$ of the infected plants per week, a difficult but not impossible goal. But if the higher estimate were correct, even if managers could achieve a $100 \%$ kill rate they would not eliminate the disease. The figures for Nicaragua seem slightly more encouraging. At the low end of the scale the 


\section{TABLE 1}

Estimates of parameters for the corn stunt Spiroplasma in Central America. Nica refers to The Santa Rosa experiment station of the Ministry of Agriculture near Managua Nicaragua, and CR refers to The Fabio Baudrit experiment station of the University of Costa Rica, near Alajuela, Costa Rica.

\begin{tabular}{llll}
\hline Parameter & Value & Site & Reference \\
\hline$\mu_{2}$ & $0.129^{\mathrm{a}}$ & Nica (1984) & Power, 1987 \\
$b$ & $1-3^{\mathrm{b}}$ & Nica (1984) & Power, unpublished data \\
$N_{2} / N_{1}$ & 0.3 & CR (1987) & Power, unpublished data \\
& 1.0 & CR (1986) & Power, unpublished data \\
& 10.0 & Nica (1982) & Power, 1988 \\
$f_{1}$ & 20 & Nica (1983) & Power, 1985 \\
$f_{2}$ & $0.24-0.68^{\mathrm{c}}$ & & (see text) \\
\hline
\end{tabular}

\footnotetext{
a Individuals leaving the plot per day.

b Plants encountered per day.

${ }^{c}$ Probabilities of infection.
}

target elimination rate for diseased maize is less than $1 \%(0.11 \%)$ while the high end reaches $8.3 \%$. A figure of $8 \%$ is well beyond practicality, but less than $1 / 2$ of $1 \%$ would probably not be difficult. As we noted earlier, in an area in which the disease is very common, such as in Nicaragua, the methodology of eliminating heavily infested fields or infected individuals should be more efficient than in areas in which the disease is rare, such as in Costa Rica, as reflected in these calculations. But to make such calculations have any real practical significance we feel that better estimates of the basic parameters are imperative. The uncertainty suggested by a range of estimates between $2 \%$ and over $100 \%$, as we calculate for Costa Rica, is large to say the least. Nevertheless, at least for the parameter estimates from Nicaragua, these preliminary data suggest that (a) the technique of removing infected maize may be viable and (b) consequently programs for estimating the necessary parameters should be encouraged.

Direct transmission form of the model. If we use the classic assumption that the population dynamics of the vector are very rapid relative to the dynamics of the corn population (i.e. both the size of the leafhopper population and the fraction of insects infected stays constant over the time period of interest), we can collapse equations (1a) and (1b) into a simple single equation by setting $\mathrm{d} l_{2} / \mathrm{d} t=0$ (May and Anderson, 1979), whence we obtain:

$$
\mathrm{d} I_{1} / \mathrm{d} t=\left[\left(N_{1} / N_{2}\right) b_{2} b_{1} I_{1}\left(1-I_{1}\right) /\left(\mu_{2}+b_{1} I_{2}\right)\right]-\mu_{1} I_{1}
$$


which is a simple description of the dynamics of infection in the corn, as it approaches its inevitable 100\%, and is, as May and Anderson (1979) point out, exactly the same as the model for a direct transmission process. Thus, equation (2) represents the simplest possible manifestation of the corn stunt disease, where the transmission process of the spiroplasma operates in the context of a constant population size and infection rate of the leafhopper. We seriously doubt that equation (2) can be used as a precise descriptor of many processes in the field, although it may be of use in investigating several qualitative phenomena.

In particular with regard to the question raised above about artificially raising the 'death rate' of infected corn by a management program of eliminating fields with high infection, a casual examination of equation (4) suggests a further qualitative conclusion which augments the original conclusion. Increasing $\mu_{1}$ not only reduces the equilibrium level of infestation, it also reduces the rate at which that equilibrium is approached.

With regard to applying the direct transmission model in a quantitative sense as a management tool, we cite only one fairly obvious result that mitigates against such a strategy. Power's data with planting densities clearly showed that the infection rate (i.e. $I_{1}$ ) of corn decreased as the corn density was increased (Power, 1987). From the direct transmission model we would expect no change at equilibrium as a function of corn planting densities, since $I_{1}$ approaches unity regardless of parameter values. In Fig. 2 we present three trajectories of equation (4), representing three corn densities, whence it is clear that at any point in time, the larger corn density will show a higher infection rate. Casual inspection of equation (4) suggests this to be a general result, since $N_{1}$ enters as a simple multiplier of the derivative. In any two systems beginning with the same rate of infection, the system with

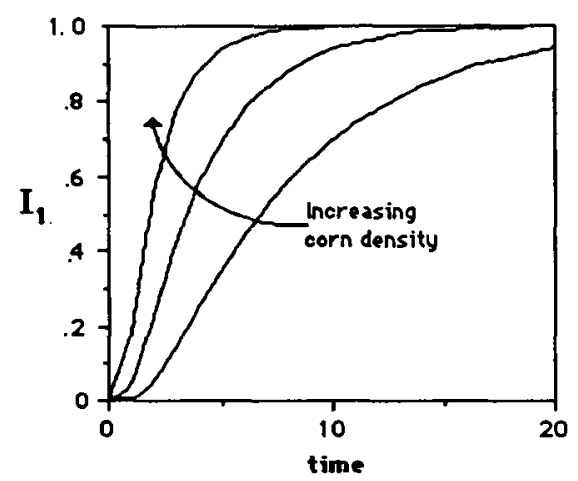

Fig. 2. Three trajectories of equation (4), illustrating different evolution of infection rate as a function of different corn densities or the direct transmission process. Base parameters are as in Fig. 1, except low $N_{1}=50$, medium $N_{1}=100$, and high $N_{1}=200$. 
the higher corn density will have the larger rate of infestation at any point in time. To rationalize Power's result in the context of the classic epidemiological model requires either that the equilibrium infection decreases with a larger population or the rate at which the proportion of attacked individuals increases itself decreases with a larger population. Neither can be true in the direct transmission process. It is seemingly not possible to mimic the qualitative nature of Power's results through the use of a direct transmission model. However, we note in passing that a tacit assumption in this model is the lack of multiple infections. If multiple infections are included as non-linear terms in the original equations it might be possible to mimic Power's empirical results at least qualitatively.

\section{DISCUSSION}

Equations (1a) and (1b) provide a first approximation of the dynamics of infection at a local level, and equation (4) provides a description of the build-up of the disease in the corn population, assuming the dynamics of the secondary host are unimportant. As we have seen, the classic direct transmission model does not appear to accurately predict infection rates when corn density is varied. The inadequacy of this configuration of the model may result from an intrinsic difference between transmission processes for animal and plant hosts. For the animal hosts that these models typically represent, increases in the host population size result in more contacts with the vector or secondary host and therefore higher infection rate. In contrast, higher plant populations do not necessarily result in more contacts with the vector, since contacts are simply a function of vector tenure time per host, seemingly a constant regardless of host density (Power, unpublished data). Of course, number of contacts and consequent infection rates should vary with changes in the vector (secondary host) population. Furthermore, the assumption that the size of the leafhopper population remains constant over a single corn generation is clearly violated since our data show significant changes due to leafhopper immigration, death, and reproduction during this period (Power, 1987, 1988).

An important difference between the classical model and our application of it here is that the corn does not recover, and an individual infected corn plant remains infectious for the rest of the season. While the conventional wisdom holds that an infected individual remains infectious as long as it remains alive, and that is the assumption on which our model is based, concrete information on the period of infectiousness is not formally available. If future investigations discover that the spiroplasm becomes inviable in stunted plants, we must conclude that the value of $\mu_{1}$ is not always very small as we have asserted. 
A limitation of the general model is that two of the most important dynamic variables are excluded from equations (1) and (2), the population densities of corn and the leafhopper (their ratio is included as a constant). Because leafhoppers are not affected by the spiroplasma and corn densities are determined by planting activities, these two variables are not responsive to the disease and thus in a sense are outside of the disease system, making the general conclusions of the present work tenable. On the other hand, the leafhopper density has a strong input into the disease system, and high versus low leafhopper densities can strongly affect the properties of the system. The corn population varies throughout the year, with three peaks of planting activity. The leafhopper population responds to increased corn density with increased growth rate, but the reverse is not true, that is, maize planting is not curtailed significantly in response to a large outbreak of the leafhopper. Thus the dynamics of the two population density variables must be included in an expanded version of the model. This work is currently in progress.

The present approach is intended to describe the dynamics of the system at a local level. But some of the most interesting ecological features of the system have to do with its spread through space. Are there local pockets of infection that then slowly diffuse to other areas? Is the primary source of infection, on the average, from long distance immigrants or do the local dynamics account for most of the local spread of the disease? These and similar questions require an approach that includes the dynamics through space (e.g. Minogue and Fry, 1983a; Van den Bosch et al. 1988a,b) in addition to the dynamics through time, also work in progress.

Ultimately a series of biological and economic issues must be considered. First, in addition to the spiroplasma, two other pathogens, vectored by the same insect are known, maize rayado fino virus (MRFV) and maize bushy stunt mycoplasma (MBSM). Both pathogens are found in Central America (Power, personal observation), and are significant problems in some places. Their potential interaction with the spiroplasma could dramatically alter the dynamics of the system, and remain to be investigated.

In terms of the practical question concerning the utility of roguing diseased plants in order to lower infection rates, there are some difficulties here as well. As noted above, it may take from 16 to 46 days for symptoms of the disease to become apparent. Both corn variety and the titer of the transmitted spiroplasma probably play a role in this incubation period variability, but the dominant cause is likely ambient temperature. In a single variety of sweet corn, Nault (1980) found an average latent period of 43 days at a day/night temperature cycle of $27 / 18^{\circ} \mathrm{C}$, but a latent period of only 16 days at a $31 / 25^{\circ} \mathrm{C}$ cycle. The higher temperature cycle is quite typical of most of the pacific coast of Central America where corn stunt is a 
severe problem. Because the variance in our estimate of the rate of roguing necessary to control corn stunt is largely based on the variance in incubation period, we expect that the lower estimates of roguing rate may be reasonable for most areas of the pacific coast. On the other hand, the lower temperature cycle may be a more reasonable estimate of conditions in the mountainous areas, implying an impractically high roguing rates for control of the disease there. Thus the strategy of selective destruction of diseased plants may be practical only where both leafhopper densities and ambient temperatures are high.

One further problem is that it is quite probable that the spiroplasma titer in an infected plant becomes sufficient for transmission before symptoms are actually expressed. This could make roguing plants an ineffective strategy for controlling corn stunt within a given field. However, strategic destruction of infected fields could be useful for regional control of corn stunt, or for controlling maize rayado fino virus which has much shorter latent periods and very fast onset of symptoms.

Corn stunt is, in some areas of Central America, the most important problem in corn production, both for subsistence farmers and large commercial growers. Its control has been thus far elusive. An understanding of the ecology and epidemiology of the system would, we feel, significantly contribute to efforts to develop effective management programs. This initial model formulation is intended as a first step in this direction.

\section{REFERENCES}

Anaya Garcia, M.A., 1975. Determinación del período mínimo y óptimo de inoculación necesaria para que el vector Dalbulus maidis transmite el patogeno causante del achaparramiento del maíz. Siades (El Salvador), 4: 9-14.

Anderson, R., 1981. Population ecology of infectious disease agents. In: R.M. May (Editor), Theoretical Ecology: Principles and Applications (2nd Edition). Sinauer, Sunderland, MA, pp. $318-355$.

Anderson, R.M. and May, R.M., 1979. Population biology of infectious diseases: part I. Nature, 280: 361-367.

Anderson, R.M. and May, R.M., 1982 (Editors), Population Biology of Infectious Diseases. Dahlem Konferenzen, 14-19 March 1982, Berlin. Springer, Berlin, 314 pp.

Aron, J.L. and May, R.M., 1982. The population dynamics of malaria. In: R.M. Anderson and R.M. May (Editors), Population Biology of Infectious Diseases. Dahlem Konferenzen, 14-19 March 1982, Berlin. Springer, Berlin, pp. 139-179.

Bailey, N.T.J., 1975. The Mathematical Theory of Infectious Diseases and its Applications (2nd Edition). Griffin, London, 413 pp.

Burdon, J.J., 1982. The effect of fungal pathogens on plant communities. In: E.I. Newman (Editor). The Plant Community as a Working Mechanism. Blackwell, Oxford, pp. 99-112

Burdon, J.J., 1987. Diseases and Plant Population Biology. Cambridge University Press. Cambridge, 208 pp. 
Cohen, J.E., 1976. Schistosomiasis: a human host-parasite system. In: R.M. May (Editor), Theoretical Ecology: Principles and Applications (1st Edition). Blackwell, Oxford, pp. 237-256.

Davis, R.E., 1974. Spiroplasma in corn stunt-infected individuals of the vector leafhopper Dalbulus maidis. Plant Dis. Rep., 58: 1109-1112.

Dinoor, A. and Eshed, N., 1984. The role and importance of pathogens in natural plant communities. Annu. Rev. Phytopathol, 22: 443-466.

Ellstrand, N.C. and Antonovics, J., 1985. Experimental studies of the evolutionary significance of sexual reproduction. II. A test of the density-dependent selection hypothesis. Evolution, 39: 657-666.

Gadea, A. and Power, A., 1984. Evaluation of the population dynamics of Dalbulus maidis, the incidence of corn stunt, and Spodoptera frugiperda damage in six monthly plantings of maize. Programa Coop. Centroam. Mejoram. Cult., 30: 21 (in Spanish).

Hamilton, W.D., 1982. Pathogens as causes of genetic diversity in their host populations. In: R.M. Anderson and R.M. May (Editors), Population Biology of Infectious Diseases. Dahlem Konferenzen, 14-19 March 1982, Berlin. Springer, Berlin, pp. 269-296.

Jeger, M.J., 1983. Analysing epidemics in time and space. Plant Pathol., 32: 5-11.

Leonard, K.J. and Czochor, R.J., 1978. Theory of genetic interactions among populations of plants and their pathogens. Annu. Rev. Phytopathol., 18: 237-258.

Liu, W., 1988. Mathematical models of infectious diseases in multiple populations. Cornell University, Ithaca, NY, 8 pp.

Lotka, A.J., 1923. Contributions to the analysis of malaria epidemiology, Am. J. Hyg. 3 (Suppl. 1), $121 \mathrm{pp}$.

Macdonald, G., 1957. The Epidemiology and Control of Malaria. Oxford University Press, London, $201 \mathrm{pp}$.

Madden, L.V., Nault, L.R., Heady, S.E. and Styer, W.E., 1984. Effect of maize stunting mollicutes on survival and fecundity of Dalbulus leafhopper vectors. Ann. Appl. Biol., 105: 431-441.

Markham, P.G. and Alivizatos, A.S., 1983. The transmission of corn stunt spiroplasma by natural and experimental vectors. In: D.T. Cordon, J.K. Knoke, L.R. Nault and R.M. Ritter (Editors), Proc. Int. Maize Virus Disease Colloquium and Workshop, 2-6 August 1982, Ohio Agricultural Research and Development Center, Wooster, OH, pp. 56-61.

Martinez-Lopez, G., 1976. New maize virus deseases in Colombia. In: L.E. Williams, D.T. Gordon, and L.R. Nault (Editors), Proc. Int. Maize Virus Disease Colloquium and Workshop, August 1976, Ohio Agricultural Research and Development Center, Wooster, OH, pp. 20-29.

May, R.M. and Anderson, R.M., 1979. Population biology of infectious diseases: part II. Nature, 280: 455-461.

Minogue, K.P. and Fry, W.E., 1983a. Models for the spread of plant disease: Model description. Phytopathology, 73: 1168-1173.

Minogue, K.P. and Fry, W.E., 1983b. Models for the spread of plant disease: Some experimental results. Phytopathology, 73: 1173-1176.

Nault, L.R., 1980. Maize bushy stunt and corn stunt: a comparison of disease symptoms, pathogen host ranges, and vectors. Phytopathology, 70: 659-662.

Nault, L.R., 1983. Origins in Mesoamerica of maize viruses and mycoplasmas and their leafhopper vectors. In: R.T. Plumb and J.M. Thresh (Editors), Plant Virus Epidemiology. Blackwell, Oxford, pp. 259-266.

Nault, L.R. and DeLong, D.M., 1980. Evidence for coevolution of leafhoppers in the genus 
Dalbulus (Cicadellidae: Homoptera) with maize and its ancestors. Ann. Entomol. Soc. Am., 73: 349-353.

Nault, L.R. and Madden, L.V., 1985. Ecological strategies of Dalbulus leafhoppers. Ecol. Entomol., 10: 57-63.

Nault, L.R., DeLong, D.M., Triplehorn, B.W., Styer, W.E. and Doebley, J.F., 1983. More on the association of Dalbulus (Homoptera: Cicadellidae) with Mexican Tripsacum (Poaceae), including the description of two new species of leafhoppers. Ann. Entomol. Soc. Am., 76: 305-309.

Power, A.G., 1985. Plant community diversity, insect movement, and the dynamics of an insect-transmitted plant disease. Ph.D. thesis, University of Washington, Seattle, WA, 146 pp.

Power, A.G., 1987. Plant community diversity, herbivore movement, and an insect-transmitted disease of maize. Ecology, 68: 1658-1669.

Power, A.G., 1988. Leafhopper response to genetically diverse maize stands. Entomol. Exp. Appl., 49: 213-219.

Ross, R., 1911. The Prevention of Malaria (2nd Edition). Murray, London, $669 \mathrm{pp}$.

Thresh, J.M., 1983. Progress curves of plant virus disease. Adv. Appl. Biol., 8: 1-85.

Van den Bosch, F., Zadoks, J.C., and Metz, J.A.J., 1988a. Focus expansion in plant disease. I: The constant rate of focus expansion. Phytopathology, 78: 54-58.

Van den Bosch, F., Zadoks, J.C., and Metz, J.A.J., 1988b. Focus expansion in plant disease. II: Realistic parameter-sparse models. Phytopathology, 78: 59-64.

Van den Bosch, F., Zadoks, J.C., and Metz, J.A.J., 1988c. Focus expansion in plant disease. III: Two experimental examples. Phytopathology, 78: 919-925.

Vanderplank, J.E., 1963. Plant Diseases: Epidemics and Control. Academic Press, New York, $349 \mathrm{pp}$.

Warren K.S. and Newill, V.A., 1967. Schistosomiasis - A Bibliography of the World's Literature from 1852 to 1962 . Western Reserve University Press, Cleveland, OH, Vols. 1, $2,598+395 \mathrm{pp}$.

Zadoks. J.C. and Schein, R.D., 1979. Epidemiology and Plant Disease Management. Oxford University Press, New York, 427 pp. 